\title{
Exploration des compétences initiales relatives au français de scolarisation chez des élèves hispanophones de $1^{\text {re }}$ année scolarisés en Outaouais
}

\author{
Marie-Pier Bastien \\ Université d'Ottawa \\ Carole Fleuret \\ Université d'Ottawa
}

\begin{abstract}
Résumé
Cette étude s'intéresse aux connaissances que détiennent les élèves hispanophones au regard du français de scolarisation, dans l'objectif de mieux comprendre les difficultés rencontrées par cette communauté. Précisément, l'étude explore les compétences initiales relatives au français langue de scolarisation de 10 élèves hispanophones scolarisés en Outaouais au regard du principe alphabétique (ex. linéarité, orientation, etc.) et du code alphabétique (ex. connaissance des lettres, des syllabes et des phonèmes). Pour en rendre compte, une recherche qualitative a été menée. Les résultats montrent que certaines tendances sont observables, notamment que l'élève hispanophone de première année peut reconnaître et identifier les lettres de l'alphabet. L'identification des allographes s'est également avérée une activité facile à réaliser. Nos résultats nous invitent aussi à décrire les fonctions du français de scolarisation chez les participants de notre étude, à savoir que l'utilité du lire-écrire réside principalement dans des activités qu'ils accomplissent au quotidien. Relativement aux aspects paratextuels et aux divers types de livres, ceux-ci semblent totalement inconnus par les élèves participants. On peut supposer que l'exposition aux livres à la maison soit plutôt rare.
\end{abstract}

\begin{abstract}
This article explores the initial French language knowledge of 10 Spanish-speaking students in the Outaouais region in terms of the alphabetic principle (i.e., linearity, orientation, etc.) and the alphabetic code (e.g., knowledge of letters, syllables, and phonemes). To account for this, qualitative research has been conducted. Results of this study show that certain tendencies are observable, in particular, that first-grade Spanish-speaking students can recognize and identify letters of the alphabet. Identifying allographs has also proven to be an easy activity. Our results also invite us to describe the French schooling functions of the participants in our study, namely that the usefulness of reading-writing lies mainly in the activities they perform on a daily basis. With regard to the paratextual aspects and the various types of books, these seem completely unknown to the participants. It can be assumed that exposure to books at home is rather rare.
\end{abstract}




\section{Exploration des compétences initiales relatives au français de scolarisation chez des élèves hispanophones de $1^{\text {re }}$ année scolarisés en Outaouais}

\section{Introduction et problématique}

En 2016, la province du Québec accueillait 14,4 \% des allophones au Canada (Statistique Canada, 2017). Nonobstant la forte croissance d'immigrants dans les grandes villes québécoises telles que Montréal et Québec, une hausse rapide de cette population a été notée à l'extérieur de la région métropolitaine. Il n'est donc pas surprenant de constater qu'entre 2011 et 2016, la région de 1'Outaouais accueillait 7535 nouveaux arrivants (Statistique Canada, 2017). Un article publié par la ville de Gatineau (2013) a précisé que les Portugais formaient la communauté d'immigrants la plus importante, mais qu'au cours des dernières années, de fortes vagues d'immigrants en provenance d'Amérique centrale et du Sud se sont installées en Outaouais.

Sur le plan langagier, on constate que la majorité des familles qui ont immigré en Outaouais sont allophones, c'est-à-dire que $68 \%$ des nouvelles arrivées ont une langue parlée autre que le français, l'anglais ou une langue des premières nations. Précisément, on recense, dans le milieu familial l'arabe, l'espagnol, le portugais, les langues chinoises, le serbo-croate et le roumain (Ville de Gatineau, 2013). Quant à la réussite scolaire, et considérant le nombre d'immigrants qui fréquentent les écoles francophones du Québec, Mc Andrew (2009) a montré que celle des allophones était équivalente à la réussite des locuteurs des langues officielles, à l'exception des matières où les composantes culturelles et linguistiques étaient omniprésentes; à titre d'exemple, on peut noter l'anglais, l'histoire et la géographie, car leurs connaissances demeurent fragiles et leur compréhension de la langue de scolarisation n'est pas encore suffisamment établie.

Pour faciliter la réussite scolaire des élèves allophones, le Québec met en place, depuis les années 1960, de nombreux programmes d'aide et de soutien. Parmi ceux-ci figurent les classes d'accueil ${ }^{i}$ et les classes de francisation ${ }^{\text {ii }}$ (Armand, 2011) qui visent à favoriser le développement d'habiletés langagières chez les élèves. Le Programme d'accueil et de soutien à l'apprentissage du français (PASAF), mis en place dans les écoles du Québec en 1997, a également permis de mieux cerner les besoins des élèves allophones et d'organiser en conséquence les différents modèles de services qui leur sont offerts. Précisément, ce sont cinq modèles, qui sont offerts aux élèves et dont l'implantation dépend de leurs besoins, allant d'une intégration en classe fermée à l'intégration totale en salle de classe.

Bien qu'il existe un certain nombre de mesures d'appui à l'apprentissage du français, on peut se questionner sur l'impact de ces dernières auprès de certaines communautés puisqu'elles ne semblent pas être suffisantes pour permettre à une majorité de décrocher un diplôme d'études secondaires. Précisément, si l'on regarde les groupes d'un point de vue ethnolinguistique, certains semblent être plus fragiles quant à la réussite scolaire, c'est-à-dire qu'ils semblent éprouver davantage de difficultés, ce qui est, entre autres, le cas des hispanophones (Armand, 2011).

\section{La communauté hispanophone}

Dans cette section, nous présenterons brièvement la communauté hispanophone de l'Outaouais de manière à mieux circonscrire ses caractéristiques et afin de souligner 
certains facteurs qui peuvent interférer sur la réussite scolaire des élèves.

La majorité des membres de la communauté d'Amérique latine qui s'est installée au Québec, et notamment en Outaouais, est née à l'extérieur du pays $(67,4 \%$ ) (Immigration et Communautés culturelles, 2006). De manière générale, ce sont des immigrants en provenance du Mexique, du Pérou, de la Colombie et de l'Uruguay ; ils forment les communautés latines les plus importantes à Gatineau (Ville de Gatineau, 2013). En ce qui concerne l'état matrimonial, plus des deux cinquièmes $(45,5 \%)$ des personnes d'origine latino-américaine sont célibataires et 39,8\% sont légalement mariées et non séparées (Immigration et Communautés culturelles, 2006). Sur le plan scolaire, 24,2 \% des LatinoAméricains détiennent une scolarité inférieure au diplôme d'études secondaires et 23,2 \% détiennent un diplôme d'études secondaires (Immigration et Communautés culturelles, 2006).

Sur le plan langagier, $84 \%$ des membres de la communauté sont de première génération (Immigration et Communautés culturelles, 2006), ce qui signifie qu'une infime partie des Latino-Américains qui résident à Gatineau sont nés ici. Si une majorité dit connaître le français ou l'anglais (88,8 \%), l'espagnol demeure la langue du groupe et de l'identité collective et cet idiome se caractérise par son oralité (Bastien et Fleuret, 2018 ; Bastien, 2017 ; Gamez et Levice, 2013; Delgado-Gaitan, 2005). Si l'on regarde maintenant le volet économique, on constate que : 1) le revenu moyen d'une famille latino-américaine est de 21539 \$, comparativement à l'ensemble de la population québécoise (32 074\$), 2) le taux de chômage est de 13,1\% par rapport à 7,0 \% dans les foyers québécois (Immigration et Communautés culturelles, 2006).

Comme nous pouvons le constater, les immigrants hispanophones vivent dans une certaine précarité économique et connaissent le chômage. Ils sont également peu scolarisés comme l'ont souligné les statistiques témoignant de leur niveau de scolarité (Immigration et Communautés culturelles, 2006). Il est reconnu, dans les écrits scientifiques, que ces facteurs peuvent influer sur la réussite scolaire (Mc Andrew et coll., 2009; Kanouté, Vatz Laaroussi, Rachédi et Tchimou Doffouchi, 2008; Dagenais, 2012). C'est pourquoi il nous apparaissait pertinent de présenter brièvement la communauté hispanophone de l'Outaouais et les caractéristiques qui lui sont inhérentes. Enfin, dans la mesure où les hispanophones sont une immigration récente dans la région de l'Outaouais, il nous semble important de nous questionner sur les connaissances que détiennent les élèves au regard du français afin de mieux comprendre les difficultés rencontrées par cette communauté.

\section{Cadre conceptuel}

Par définition, le concept de langue étrangère se construit par opposition à celui de la langue d'origine (L1) ${ }^{\text {iii. }}$. Selon Cuq (2003, p.159), « toute langue non maternelle est une langue étrangère à partir du moment où elle représente, pour un individu ou un groupe, un savoir encore ignoré, une potentialité, un objet nouveau d'apprentissage. » Si plusieurs chercheurs se sont penchés sur l'apprentissage de la langue seconde (L2) en contexte scolaire, Cummins (1979) a fait émerger l'hypothèse de l'interdépendance qui montre qu'en contexte scolaire, il existe une relation tangible entre la L1 et la L2 lorsqu'il s'agit de développer des connaissances linguistiques, car il y a l'existence d'un transfert. Il a mentionné également que le niveau atteint en L2 dépendait considérablement du niveau atteint en L1. Toutefois, le chercheur a noté que l'apprenant devait avoir atteint un certain 
seuil de connaissances initiales dans sa langue d'origine afin que celui-ci puisse développer un certain niveau linguistique dans la langue seconde.

À l'instar des propos de Cummins, ceux de Baker (2001) font référence à quatre avantages qui sont relatifs aux transferts linguistiques, à savoir 1) que l'action de traiter de l'information dans deux langues peut mener à une compréhension plus approfondie d'un concept; 2) que l'apprentissage de la langue seconde peut agir en tant que support à l'apprentissage de la langue d'origine; 3) que l'apprentissage d'une langue seconde se fait à l'intérieur et à l'extérieur de l'enceinte scolaire; et 4) que l'intégration de l'apprenant en langue seconde se fait plus facilement lorsque celui-ci baigne dans un environnement où la L1 et la L2 se chevauchent.

Auger (2007), à la suite d'une recherche de 13 mois menée auprès d'élèves nouvellement arrivés en France, a avancé que l'enseignement explicite dans la L1 influençait positivement l'apprentissage de la L2, dans la mesure où l'élève est amené à réfléchir sur ses référents dans sa langue d'origine. Inversement, elle a mentionné que l'absence d'enseignement de la langue d'origine pouvait rendre difficile l'apprentissage et l'acquisition de la L2, en supposant que les élèves n'ont pas nécessairement développé la littératie dans leur L1 et qu'ils se confrontent au double défi de développer ces compétences à l'écrit dans une langue seconde. Pour Auger, ce sont donc les allers-retours cognitifs entre la L1 et la L2 qui permettraient à l'élève d'acquérir plus facilement des connaissances relatives à la langue de scolarisation, en supposant que celle-ci est une langue seconde.

En Ontario, dans une étude qui s'est intéressée à l'appropriation de l'écrit lors de résolution de problèmes orthographiques, Fleuret (2013) a également montré que, lorsqu'ils se sentent autorisés à utiliser leur L1, les élèves ont recours au transfert linguistique et font référence au métalangage dans leur L1. Durant son intervention de neuf semaines dans une classe-ressource ottavienne, l'auteure a montré que l'utilisation de la L1 en contexte de L2 permettait une certaine distanciation sur la langue de scolarisation, facilitant par le fait même son apprentissage, car les élèves étaient davantage centrés sur les propriétés du signifiant écrit que sur le signifié.

L'importance de la L1 dans l'acquisition de la L2 est aussi mise de l'avant dans les travaux de Hornberger (2006). Précisément, le modèle que l'auteure a proposé à la suite d'une étude menée auprès de populations indigènes suggère que l'utilisation de la langue d'origine (quechua ou maori) dans l'appropriation de la langue seconde médiatise le dialogue, permet la création de sens, offre l'accès à des discours plus larges et permet aux apprenants de prendre parole dans leur apprentissage. Le recours à leur langue, autorisé et encouragé en salle de classe, permet non seulement de favoriser l'apprentissage de la langue seconde, mais également de promouvoir le maintien et la revitalisation de leur langue d'origine.

Finalement, Castelloti et Moore (2010,p.17) ont relaté que « le simple fait de permettre aux élèves de mobiliser leurs capacités dans plusieurs langues à la fois, voire d'encourager ces productions croisées, les engage dans la communication scolaire et les rend confiants dans leurs capacités à s'investir dans la langue de scolarisation. » Mc Andrew et Ciceri (2003), quant à elles, ont mentionné que la substitution de la langue d'origine par la langue d'accueil risquait d'avoir des conséquences négatives.

En somme, nous comprenons que l'apprentissage d'une langue seconde ne se fait pas au détriment de la langue d'origine. À l'inverse, les recherches de Cummins (1979, 1981) semblent plutôt montrer que les transferts linguistiques entre la L1 et la L2 sont nécessaires à l'acquisition et l'appropriation de la langue seconde. Dans la majorité des cas, on dit 
également que cette même langue seconde est aussi une langue de scolarisation, concept que nous aborderons dans la prochaine section.

\section{La langue de scolarisation}

Par définition, la langue de scolarisation est celle apprise à l'école et pour l'école (Verdelhan-Bourgade, 2002). Considérant cette précision, on dit que pour l'apprenant en langue seconde, celle-ci n'est pas uniquement une langue « à apprendre » et qu'elle remplit plusieurs fonctions. Pour Verdelhan-Bourgade, la langue de scolarisation a 1) une fonction d'exposition du savoir - elle permet à un enseignant d'exposer la langue et de présenter des connaissances relatives à cette langue; 2) une fonction de concentration du savoir plusieurs de ses éléments concentrent le savoir; 3) une fonction instrumentale de construction du savoir - elle accompagne et rend possible le travail de la pensée qui soutient l'appropriation des connaissances et 4) une fonction d'exercisation - elle demande un certain entraînement afin de l'acquérir.

D'entrée de jeu, cette langue de scolarisation est également une langue de promotion sociale qui jouit d'une position dominante, en fonction de son statut sociolinguistique, par rapport à la langue de la famille. (Spaëth, 2008). Les langues parlées à la maison n'ont vraisemblablement pas les mêmes portées sociales et sont souvent dévalorisées dans l'enceinte scolaire. Sachant que les pratiques langagières sont indéniablement des pratiques sociales, plusieurs auteurs (Galigani, 2015 ; Spaëth, 2008 ; Le Ferrec, 2008) ont fait mention de l'importance de considérer la valeur des langues, notamment en considérant les dimensions sociales et culturelles de l'écrit.

Pour favoriser son apprentissage, Verdelhan $(1999$, p.5) a avancé que « le français de scolarisation ne doit pas être considéré comme une langue spécifiquement scolaire qui serait coupée de la vie et de la société. » Selon elle, la langue de scolarisation appartient aussi à la société et fait même partie intégrante de la culture. Ainsi, pour assurer une transmission adéquate des savoirs de la culture, celle-ci a mentionné l'importance de présenter aux apprenants des genres textuels qui leur sont propres, c'est-à-dire qui correspondent à leurs champs d'intérêt et à leurs habitudes de vie.

De son côté, Le Ferrec (2012) a mis l'accent sur l'importance de l'apprentissage explicite des composantes de la langue de scolarisation, "soit parce que cette variété est une L2 pour les élèves, soit parce qu'elle est trop éloignée des pratiques langagières des élèves et que ceux-ci ne parviennent pas à entrer dans la culture scolaire [...]. » Aussi l'auteure a-t-elle fait mention de la complexité de l'appropriation à l'écrit dans un contexte de langue de scolarisation, car celle-ci est à la fois un objet d'étude et une langue d'enseignement, sans oublier les statuts affectifs ou sociolinguistiques qui peuvent également rendre difficile l'apprentissage de la langue de scolarisation.

Pour favoriser l'apprentissage de la langue de scolarisation, et particulièrement l'entrée dans l'écrit, plusieurs études ont montré l'importance de développer les capacités métalinguistiques. En effet, la lecture étant une tâche linguistique formelle, « son apprentissage nécessite de la part de l'enfant de développer une conscience explicite des structures linguistiques qui devront être intentionnellement manipulées. » (Gombert, 1990, p.2) 


\section{Les capacités métalinguistiques}

Les capacités métalinguistiques ${ }^{\mathrm{iv}}$ font référence aux habiletés à manipuler différentes unités du langage, tant en production qu'en réception (Gombert, 1996). Précisément, « une activité métalinguistique est une activité consciente sur une ou des unités linguistiques [...] qui implique, au-delà d'une connaissance enseignée, un savoir construit qui vise, par exemple, dans un processus d'écriture à corriger un phonogramme à caractère multigraphémique. » (Fleuret, 2008, p.71)

Bialystok et Ryan, dans une recherche de 1985, se sont penchées sur l'acquisition des capacités métalinguistiques d'apprenants en langue seconde. Leur modèle a apporté un éclairage indéniable sur le développement de telles capacités en langue seconde. Dans leur modèle, on remarque l'existence de deux processus cognitifs qui se développent de manière parallèle : les connaissances analysées (analyzed knowledge) et le contrôle cognitif (cognitive control). Si les connaissances analysées font appel aux représentations symboliques de la langue que se fait l'apprenant (l'enfant passe d'une représentation implicite à une représentation explicite de la langue), le contrôle cognitif fait plutôt référence aux fonctions et au contrôle qui sont impliqués dans des tâches linguistiques (capacité à focaliser son attention sur des informations pertinentes de la tâche linguistique). Les auteures ont mentionné que ces deux processus opéraient de façon parallèle au fur et à mesure que l'enfant développait et «maîtrisait » le langage. Concrètement, pour Bialystok et Ryan (1985), plus l'apprenant développe ses habiletés dans une langue, moins le niveau de contrôle qu'il demande est élevé. Comme l'a expliqué Fleuret (2008, p.126), en reprenant les conclusions de Bialystok et Ryan: « l'automaticité détermine fortement le niveau de contrôle demandé dans l'activité à réaliser. » Nous comprenons donc que ce sont ces deux processus qui permettent à l'enfant de passer d'un langage conventionnel à un langage plus précis, à force de parler, de lire et d'écrire.

Si les capacités métalinguistiques englobent à la fois les connaissances métaphonologiques, morphologiques, syntaxiques et sémantiques, notons que nous focaliserons uniquement sur les capacités métaphonologiques puisque de nombreux travaux tant en L1 qu'en L2 (Gombert, 1992; Armand, 2005; Fleuret et Montésinos-Gelet, 2011) mentionnent une forte corrélation entre ces dernières et la réussite en lecture.

\section{Les capacités métaphonologiques}

Si nombreuses soient les études qui établissent des liens entre l'apprentissage de la lecture et les capacités métalinguistiques, c'est parce que la socialisation à l'écrit se fait initialement en établissant les composants phonologiques des unités linguistiques. Gombert (1990) a mentionné justement que certains travaux montraient l'importance de la conscience phonologique dans les débuts de l'apprentissage de la lecture. Précisément, celui-ci (p.3) a expliqué que « ces travaux montrent l'existence de liens entre l'apprentissage de la lecture et la possibilité d'effectuer avec succès diverses tâches métalinguistiques de manipulation de certains aspects phonologiques du langage oral. » Ces liens sembleraient être de deux sortes, à savoir 1) le contact avec l'écrit provoque l'apparition des capacités métaphonologiques, et 2) les capacités métaphonologiques facilitent 1'apprentissage de la lecture.

Aujourd'hui, plusieurs auteurs (Ouellette et Beers, 2010; Melby-Lervag, Hallaas

Lyster et Hulme, 2012), pour n'en citer que quelques-uns, affirment des relations de 
causalité réciproque entre ces capacités et l'apprentissage de la lecture. En langue seconde, plusieurs auteurs se sont penchés sur les liens qu'entretiennent les capacités métalinguistiques et la lecture. Parmi ceux-ci, Valdois (2010) a montré que la lecture permettrait à l'élève de segmenter différentes composantes de la chaîne sonore telles que les phonèmes et les syllabes de manière à en développer des capacités métaphonologiques.

Dans la même veine, en langue seconde, l'étude de cas multiples exploratoire de Doa (2013) menée auprès d'élèves arabophones du préscolaire scolarisés au Québec a permis de mettre en relation l'effet de l'âge sur le développement orthographique et sur le transfert langagier. Brièvement, les résultats obtenus par Doa (2013) indiquent que les résultats des élèves arabophones sont meilleurs lorsque les apprenants font appel aux transferts langagiers, ceux-ci développant de meilleures habiletés sur le plan métaphonologique et facilitant par le fait même l'apprentissage du lire-écrire.

À l'instar de Dao (2013), Armand, Sirois et Abadou (2008) se sont intéressés à la relation existante entre les capacités métaphonologiques et les habiletés de prélecture en français langue seconde chez des élèves d'origine immigrante du préscolaire. Dans une recherche réalisée en milieu montréalais plurilingue et défavorisé, les auteurs ont montré qu'il existe en fait une corrélation entre les capacités métaphonologiques et les habiletés en prélecture au préscolaire. Par ailleurs, leur étude a montré que les activités d'Éveil aux langues et que la reconnaissance et la légitimation des langues d'origine des enfants allophones permettent aux apprenants de développer des connaissances et des savoir-faire plus assis dans la L2.

Au regard de la population qui nous intéresse, Vernon et Ferreiro (1999) se sont intéressés à la relation entre la conscience phonologique et le développement de l'écriture chez des enfants hispanophones de maternelle scolarisés aux États-Unis. Leur étude a montré que la capacité des enfants à gérer les tâches de segmentation orale dépend grandement de leur connaissance des lettres et du système d'écriture. En proposant plusieurs implications pédagogiques au regard de leurs conclusions, les auteures ont mentionné surtout que la conscience phonologique était une condition préalable pour apprendre à lire et à écrire.

Vernon, Calderón et Castro (2004) ont montré dans une recherche menée auprès de 100 élèves mexicains, dans leur langue d'origine, qu'il existe une corrélation significative entre la conscience phonologique et le développement de l'écriture. Concrètement, les élèves de leur étude étaient soumis à deux tâches d'écriture de suppression du phonème initial ; la première tâche consistait à un recevoir un stimulus oral et la deuxième tâche consistait à recevoir à la fois un stimulus oral et l'écriture du mot. Les résultats de leur recherche montrent que l'élève parvient plus facilement à résoudre la tâche de suppression du phonème lorsqu'il reçoit une tâche écrite.

Finalement, pour leur part, López et Greenfield (2004) se sont intéressés à l'effet des capacités métaphonologiques en anglais et en espagnol de 100 élèves hispanophones scolarisés aux États-Unis sur la maîtrise de l'anglais langue seconde. À l'aide d'une régression multiple hiérarchique, les auteurs ont suggéré que les compétences métalinguistiques et métaphonologistiques des apprenants devraient être renforcées parce qu'elles sont susceptibles de faciliter leur apprentissage de l'anglais en tant que L2.

À la lumière de ce qui précède, nous comprenons que les tâches de conscience phonologique qui demandent aux élèves, par exemple, de dénombrer, de soustraire, d'ajouter ou de manipuler des phonèmes dans une unité linguistique sont essentielles au développement de la compétence en lecture. Gombert (1990) a expliqué que l'inverse est 
vrai aussi, à savoir que la difficulté à exécuter ces tâches peut poser certains défis en lecture chez l'apprenant, notamment vis-à-vis du décodage. Précisément, puisque le décodage consiste à transformer des unités orthographiques en unités phonologiques, il est démontré que la compréhension des lettres qui constituent les mots soit nécessaire.

\section{La connaissance des lettres}

Dans le même ordre d'idées, et afin d'expliquer la compréhension du principe alphabétique, Bara, Gentaz et Colé (2008) ont mentionné le lien soutenu entre la conscience phonologique et la reconnaissance des lettres. Précisément, et relativement à l'appréhension du français, ces deux compétences seraient à la base des capacités de décodage du lecteur en L1. Puisque le décodage consiste à transformer des unités orthographiques en unités phonologiques, il est démontré que la compréhension des lettres qui constituent les mots soit nécessaire. À l'inverse, on constate que certains élèves éprouvent de la difficulté en lecture, notamment en lien avec le principe alphabétique, puisqu'ils connaissent peu de lettres (De Jong et Olson, 2004).

Prévost et Morin (2015) ont avancé trois contributions à la connaissance des lettres, soit l'apprentissage du son des lettres, le développement de la conscience phonémique et l'établissement de relations lettres-sons. Parallèlement aux conclusions de Biot-Chevrier, Écalle et Magnan (2008), les auteures ont mentionné que ces trois éléments sont des prédicteurs importants des performances en lecture et en écriture. Toutefois, elles ont aussi fait mention d'un certain écart entre leurs résultats et ceux obtenus par Biot-Chevrier et coll. (2008) relatif à la connaissance des lettres et les performances en écriture des élèves, à savoir qu'elles ont noté quelques cas de dissociations entre la connaissance des lettres et les performances en écriture de certains élèves de leur étude. À ce sujet, Prévost et Morin (2015, p.43) ont défendu que la sensibilité phonologique des enfants puisse être influencée, entre autres, par « les différentes situations langagières à l'oral et à l'écrit vécues au cours de la maternelle [...]. » Pour ces chercheures, et tout comme l'a proposé Lahire (1995), cette connaissance n'est pas suffisante pour assurer le succès de l'enfant en lecture et en écriture. Plusieurs autres habiletés et connaissances sont nécessaires à l'apprentissage du lire-écrire, entre autres, la situation langagière et l'environnement familial (Prévost et Morin, 2015).

Au regard de la connaissance des lettres, en langue seconde, Bangou, Fleuret et Thibeault (2012) ont mentionné que « le développement des capacités métalinguistiques est fondamental dans l'apprentissage de la langue de scolarisation » (p.3) et que son apprentissage requiert, entre autres, un enseignement explicite. En langue seconde, on suggère également que les registres langagiers de l'élève soient considérés et que l'on autorise l'utilisation des répertoires plurilingues (Bangou et coll., 2012) comme fondement à l'apprentissage du français de scolarisation. En effet, en prenant appui sur son bagage linguistique, l'enfant peut réaliser des allers-retours cognitifs qui lui permettent de transférer ses connaissances à la L2, notamment, et dans le cas qui nous intéresse ici, en ce qui a trait à la conscience phonologique. C'est d'ailleurs pourquoi, dans cette recherche, la connaissance des lettres, cet élément que nous considérons comme central dans l'appréhension du français de scolarisation des élèves, sera explorée. 


\section{La socialisation à l'écrit}

Ce que nous avons souligné jusqu'à maintenant montre que l'appropriation de l'écrit renvoie toujours à une construction sociale, à un contexte socioculturel donné, en d'autres mots à - la socialisation à l'écrit.

Par définition, la socialisation langagière a été développée pour décrire le processus par lequel l'apprenant s'intègre à son environnement, en prenant en considération à la fois socialisation par la langue et socialisation requise dans l'utilisation de la langue (Roberts, Grandcolas et Arditty, 1999). De Pietro (2002, p.48) a signalé que le langage « participe largement à la construction sociale de la réalité et des formes de sociabilité » et que deux éléments sont à la base de cette socialisation langagière, à savoir le lieu où se déroule le discours et l'identité avec l'altérité.

Dans la même veine, Lahire (1995, p.31) a mentionné que « la structure et la forme du comportement d'un individu dépendent de la structure de ses relations avec les autres individus. » Il a aussi souligné que l'identité sociale de l'enfant se construit notamment à travers les parents. Par exemple, le fait de voir ses parents lire le journal, écrire avec aisance, organiser un calendrier ou planifier des activités à l'aide de référents visuels est des aspects naturels qui peuvent contribuer au développement littéracique de l'enfant. Toutefois, et nonobstant le comportement des parents, l'auteur a mentionné qu'il ne faut pas omettre de considérer l'éthos d'un élève, sa représentation des schèmes ainsi que ses démarches cognitives et comportementales au sein de son apprentissage afin d'envisager la pluralité des styles de réussite scolaire.

En supposant que le langage est en partie une pratique sociale, rappelons que les hispanophones ont davantage une culture langagière orale (Delgado-Gaitan, 2014). De plus, leur niveau de scolarité est faible ce qui nous amène à penser que leur rapport à l'écrit peut être fragile et qu'ils détiennent peu de familiarité avec l'écrit. Au sujet de l'objet langue, la socialisation à l'écrit peut aussi être peu connue puisque, rappelons-le, les pratiques discursives chez les hispanophones sont plutôt oralisées (Fleuret, 2008), ce qui, par ricochet, peut les conduire à avoir des connaissances restreintes des buts et des fonctions de la langue écrite. Considérant qu'il existe des dissonances entre les pratiques discursives familiales et scolaires des élèves hispanophones, on peut prétendre que la réussite scolaire ne se fait pas toujours aisément. À cet égard, Dagenais (2008, p.351) a mentionné que les styles discursifs scolaires bénéficient surtout au groupe dominant et que les groupes minoritaires semblent être « marginalisés et en danger d'échec scolaire quand leurs pratiques langagières et leurs connaissances culturelles ne sont pas soutenues par l'école. "

Au regard de ce que nous venons d'énoncer, et comme la socialisation à l'écrit des hispanophones est peu documentée en Outaouais, il nous semble important de décrire les connaissances initiales des élèves au regard du français de scolarisation pour tenter de mieux saisir les difficultés potentielles qu'ils rencontrent au regard de leur appréhension du français écrit. Dans cet ordre d'idées, notre objectif spécifique est de décrire les connaissances initiales d'élèves hispanophones de première année relatives relatives à la langue écrite, de manière à mieux comprendre leur appropriation du français langue de scolarisation. 


\section{Méthodologie}

Considérant que nous cherchons à décrire les connaissances initiales des élèves au regard du français de scolarisation, nous avons mené une recherche de type qualitative puisque celle-ci permet d'analyser des données descriptives et surtout puisqu'elle renvoie à une méthode de recherche qui s'intéresse à l'observation d'un phénomène en milieu social et naturel (Karsenti et Savoie-Zajc, 2011). Ce choix réside surtout dans son modèle interprétatif mettant l'accent sur des processus environnementaux et sociaux (Karsenti et Savoie-Zajc, 2011).

Une étude pilote a été réalisée pour nous permettre d'ajuster nos instruments, notamment pour la compréhension des énoncés. Ainsi avons-nous rencontré un élève de $1^{\text {re }}$ année n'étant pas inclus dans le projet ainsi que pour effectuer les différentes passations (entrevue, questionnaire, livret pour l'élève). À la suite de cette étude pilote, nous avons réajusté certaines questions du livret pour l'élève et modifié le contenu de certaines questions de l'entrevue pour les rendre plus accessibles quant à leur compréhension. Et en ciblant mieux nos objectifs de recherche.

\section{Participants}

Notre étude exploratoire s'est intéressée aux connaissances initiales de 10 sujets hispanophones scolarisés en Outaouais, en première année, à la Commission scolaire des Portages-de-l'Outaouais (CSPO). Le choix de cette commission scolaire repose sur un nombre significatif d'élèves hispanophones au sein de la communauté scolaire. Bien que le nombre de nos participants ne soit pas exhaustif, car nous n'avions pas le nombre total d'hispanophones fréquentant la commission, nous souhaitions avoir un nombre assez élevé d'élèves pour pallier, le cas échéant, la mortalité de l'échantillon.

Afin de solliciter les élèves et leurs parents pour cette recherche, nous avons, dans un premier temps, obtenu l'approbation du Comité éthique de l'Université d'Ottawa pour mener notre projet. Au moment de l'étude, les sujets devaient être inscrits en première année, scolarisés en français et avoir déclaré l'espagnol comme langue d'origine.

\section{Portrait des élèves}

L'âge moyen de nos dix sujets était de 6:4 ans au moment de notre étude ; il y avait cinq filles et cinq garçons. Selon la direction d'école, et en fonction du dossier scolaire des élèves, leur langue d'origine était l'espagnol, ou du moins, l'espagnol était l'une des langues d'origine déclarées. Relativement à la connaissance d'une langue autre que la langue d'origine, quatre parents ont mentionné que leur enfant connaissait le français et l'anglais, deux autres ont précisé l'anglais et deux autres encore ont mentionné que leur enfant connaissait l'espagnol. Ici, nous constatons que huit élèves sur dix connaissaient à la fois l'espagnol, le français et l'anglais, à des degrés toutefois différents. 
Tableau 1

Profil des élèves de notre étude

\begin{tabular}{lccll}
\multicolumn{1}{c}{$\begin{array}{c}\text { Nom de } \\
\text { l'élève }\end{array}$} & $\begin{array}{c}\text { Âge de } \\
\text { l'élève }\end{array}$ & $\begin{array}{c}\text { Sexe de } \\
\text { l'élève } \\
1=\text { féminin } \\
2=\text { masculin }\end{array}$ & Langue(s) d'origine & $\begin{array}{c}\text { Autre(s) langue(s) } \\
\text { connue(s) }\end{array}$ \\
\hline Sabela & 7 & 1 & Espagnol & Anglais et français \\
\hline Ronaldo & 7 & 2 & Espagnol & Anglais et français \\
\hline Tamara & 6 & 1 & Français & Espagnol \\
\hline Soraya & 6 & 1 & Espagnol & Manquant \\
\hline Alejandro & 6 & 2 & Espagnol & Anglais et français \\
\hline Herminia & 6 & 1 & Anglais & Espagnol et français \\
\hline Darío & 5 & 2 & Espagnol & Anglais et français \\
\hline Ignacio & 7 & 2 & Espagnol et français & Anglais \\
\hline Amal & 7 & 2 & Espagnol et français & Anglais \\
\hline Anabel & 7 & 1 & Espagnol et anglais & Français \\
\hline
\end{tabular}

\section{Instruments}

Pour rendre compte des connaissances initiales des élèves, nous leur avons fait remplir un livret (inspiré de la recherche de Montesinos-Gelet et Armand, 2000) dans lequel nous avons collecté des données relatives à leurs connaissances du français écrit de scolarisation (lettres de l'alphabet, syllabe, conventions du français écrit, etc.). Un entretien semi-dirigé a été réalisé en vue de finaliser notre exploration de leurs connaissances et pour nous permettre aussi de trianguler nos données.

\section{Livret sur les connaissances initiales en français de scolarisation}

Les participants ont été rencontrés individuellement, dans leur établissement scolaire, pour leur faire remplir le livret rendant compte du principe et du code alphabétique (linéarité de l'écriture, sens de la lecture, allographes, lettres, syllabes, etc.). Chaque page du livret rendait compte d'un aspect alphabétique que nous avons traité dans le cadre conceptuel qui se présente comme suit :

\begin{tabular}{|l|l|l|}
\hline Page(s) & Connaissance & Description \\
\hline 1 & $\begin{array}{l}\text { Identification des } \\
\text { lettres }\end{array}$ & $\begin{array}{l}\text { Sur papier, un éléphant est imprimé et onze éléments sont } \\
\text { inscrits à l'intérieur. On y retrouve sept lettres, à savoir les } \\
\text { lettres M, A, E, Z, X et T (2 fois) ainsi que } 3 \text { chiffres, les } \\
\text { chiffres 2, 3 et 5. L'élève doit colorier les sept lettres qui } \\
\text { figurent sur l'éléphant. }\end{array}$ \\
\hline 2 & $\begin{array}{l}\text { Reconnaissance } \\
\text { des lettres }\end{array}$ & $\begin{array}{l}\text { Nous présentons une activité sur laquelle il y a six (6) } \\
\text { ballons. Pour chaque ballon, une lettre est écrite au-dessus } \\
\text { et trois lettres sont écrites à l'intérieur. Parmi les trois lettres } \\
\text { écrites à l'intérieur des ballons, une seule est identique à } \\
\text { celle écrite au-dessus du ballon. L'enfant doit identifier la } \\
\text { lettre qui est écrite au-dessus du ballon et colorier la même } \\
\text { lettre qui est écrite à l'intérieur du ballon. }\end{array}$ \\
\hline
\end{tabular}




\begin{tabular}{|c|c|c|}
\hline 3 & $\begin{array}{l}\text { Identification des } \\
\text { allographes }\end{array}$ & $\begin{array}{l}\text { L'enfant doit entourer toutes les lettres qui sont pareilles en } \\
\text { reliant la lettre majuscule à sa lettre minuscule. } \\
\text { Précisément, } 28 \text { lettres sont écrites sur la page allouée à } \\
\text { cette activité, dont } 14 \text { sont majuscules et } 14 \text { sont } \\
\text { minuscules. Treize combinaisons sont possibles, ce qui } \\
\text { signifie que seules deux lettres se retrouveront à la fin de } \\
\text { l'activité. }\end{array}$ \\
\hline 4 & $\begin{array}{l}\text { Identification des } \\
\text { syllabes }\end{array}$ & $\begin{array}{l}\text { Nous présentons aux élèves une activité sur laquelle des } \\
\text { lettres, des syllabes et des mots sont écrits. Les élèves } \\
\text { doivent correctement entourer les } 17 \text { syllabes présentées } \\
\text { sur la feuille. }\end{array}$ \\
\hline 5 & $\begin{array}{l}\text { Connaissances } \\
\text { paratextuelles }\end{array}$ & $\begin{array}{l}\text { Ici, nous cherchons à observer les connaissances des élèves } \\
\text { à pouvoir identifier sur une première de couverture, le titre, } \\
\text { l'auteur, l'illustrateur et la maison d'édition. Pour ce faire, } \\
\text { nous leur présentons une activité sur laquelle la première de } \\
\text { couverture d'un livre est imprimé, le livre Je suis Louna et } \\
\text { je suis une artiste. Les élèves doivent correctement relier les } \\
\text { quatre éléments à l'endroit où ils se trouvent sur la page } \\
\text { couverture. }\end{array}$ \\
\hline 6 et 7 & $\begin{array}{l}\text { Orientation de la } \\
\text { lecture }\end{array}$ & $\begin{array}{l}\text { À la page } 6 \text {, nous présentons aux élèves une page du livre } \\
\text { Je suis Louna et je suis une artiste et les élèves doivent, avec } \\
\text { leur doigt, nous montrer dans quel sens se fait la lecture. La } \\
\text { page } 7 \text { diffère légèrement de la page } 6 \text { dans la mesure où les } \\
\text { élèves doivent identifier la direction dans laquelle se fait la } \\
\text { lecture lorsqu'il y a deux pages. }\end{array}$ \\
\hline 8 & $\begin{array}{l}\text { Identification des } \\
\text { mots en français }\end{array}$ & $\begin{array}{l}\text { En détail, nous présentons aux élèves une série de } 14 \text { mots } \\
\text { dont seulement } 7 \text { sont écrits en français. Les autres sont } \\
\text { écrits, par exemple, en arabe, en chinois et même en } \\
\text { japonais. Les élèves doivent entourer l'ensemble des mots } \\
\text { qui sont écrits en français. }\end{array}$ \\
\hline 9 & $\begin{array}{l}\text { Identification des } \\
\text { mots en espagnol }\end{array}$ & $\begin{array}{l}9 \text { mots sont inscrits dans un poisson. } 4 \text { mots sont écrits en } \\
\text { français (maison, lion, pomme et bleu) et } 5 \text { mots sont écrits } \\
\text { en espagnol (abuela, leche, Holà, ojos et mañana). Les } \\
\text { élèves doivent correctement entourer les mots qui sont } \\
\text { écrits en espagnol. }\end{array}$ \\
\hline
\end{tabular}

Afin d'assurer le complément du livret avec les enfants, nous les avons rencontrés dans leur établissement scolaire. Les passations, d'une durée moyenne de 30 minutes, se sont toutes déroulées dans un local, soit dans le bureau de la direction ou dans le bureau de l'orthopédagogue. Enfin, lors des passations, pour recueillir le plus d'informations possibles pouvant nous être précieuses quant au travail cognitif exercé par l'enfant, nous avions en notre possession un cahier similaire dans lequel nous avons noté les observations (subvocalisations, pauses de l'élève dans le processus d'écriture, etc.). Pour assurer l'analyse et le codage de notre étude, les réponses fournies par les élèves lors de leur passation ont été reportées dans une grille prévue à cet effet. Chaque bonne réponse était associée à un score, soit celui de 1 point. Pour toutes autres réponses, la note 0 a été attribuée. Le total 
des points a ensuite été compilé afin d'obtenir le résultat de l'élève.

Entretien semi-dirigé. Nous avons mené des entretiens semi-dirigés avec les élèves pour connaître leurs connaissances des buts et des fonctions de la langue écrite. Plus précisément, nous leur avons posé trois questions, à savoir : 1) À quoi ça sert de lire ; 2) À quoi ça sert d'écrire ; et 3) Connais-tu différents albums ? Les entretiens ont été tenus après la passation du livret. Les entretiens ont tous été enregistrés et nous les avons ensuite retranscrits sous forme de verbatim. De notre côté, nos observations ont complété la production de chaque élève afin d'avoir le portrait le plus juste possible de sa connaissance du code alphabétique et des buts et fonctions de la langue. Les réponses fournies ont été segmentées en unité de sens, qui ont été à leur tour codées et entrées dans un second tableur Excel, en fonction des concepts clés retenus dans notre cadre conceptuel (lettres, phonèmes, syllabes, etc.).

\section{Résultats et discussion}

Dans cette section, nous présenterons et discuterons les résultats du livret et de l'entretien semi-dirigé avec les élèves. Avant cela, il convient de mentionner que les prénoms utilisés sont fictifs pour préserver l'anonymat des participants.

\section{Code alphabétique}

Relativement au code alphabétique, nous nous intéressions aux connaissances initiales des élèves au regard de la discrimination visuelle, des lettres, des allographes et des syllabes, et cherchions à décrire leurs connaissances paratextuelles.

Discrimination visuelle et reconnaissance des lettres. Les premières pages du livret destiné à l'enfant portaient sur la discrimination visuelle et sur l'identification des lettres. Précisément, les élèves devaient reconnaître les lettres de l'alphabet parmi plusieurs éléments (lettres, chiffres, symboles) et les nommer.

Tous les enfants de notre étude ont su reconnaître et nommer les douze lettres qui leur étaient présentées dans cette activité. Si nous savons que la connaissance des lettres permet, entre autres facteurs, à mieux appréhender la lecture (De Jong et Olson, 2004), on peut supposer que les élèves de notre étude détiennent une base suffisante pour décoder certains messages. Comme nous savons toutefois que cette composante n'est pas suffisante pour assurer le succès de l'enfant (Lahire, 1995), nous avons cru bon de nous intéresser également à la connaissance des allographes et des syllabes.

Reconnaissance des allographes. La deuxième activité (figure 1) portait sur la reconnaissance et l'identification des allographes, c'est-à-dire, dans notre cas, sur l'identification de la lettre majuscule et de la minuscule. L'enfant devait relier la lettre minuscule à sa lettre majuscule. De manière générale $(8 / 10)$, les élèves ont su reconnaître et identifier les allographes. Les deux élèves qui ont obtenu un score moins élevé à cette activité ont confondu les lettres $b$ et $d$. Sur un plan perceptuel, on peut penser ici que la proximité visuelle des deux lettres a entrainé une confusion chez deux élèves. 


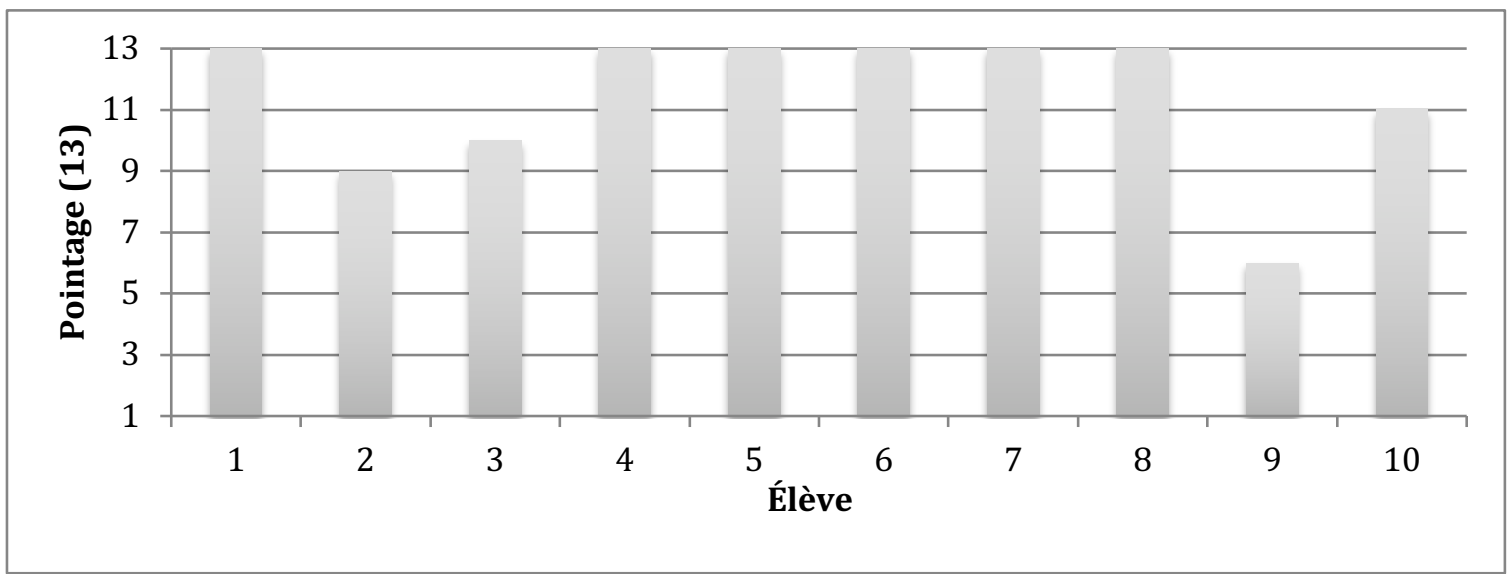

Figure 1. Score des élèves relativement à la reconnaissance des allographes.

\section{Reconnaissance des syllabes}

En ce qui concerne l'identification des syllabes, nous avons présenté aux élèves une activité sur laquelle des lettres, des syllabes et des mots étaient écrits. Les élèves, afin d'obtenir le score de 10 points, devaient discriminer visuellement et entourer les syllabes présentées sur la feuille.

Dans cette activité, le score des enfants oscille considérablement et nous remarquons des résultats très variables (figure 2). Si deux élèves ont su quasi totalement identifier les syllabes dans cette activité sans encercler autre chose, on observe, chez plusieurs élèves, ayant obtenu un score élevé qu'ils ne savent pas réellement identifier la syllabe puisqu'ils ont aussi encerclé certaines lettres en plus des syllabes. Ainsi, nous observons qu'en première année, les élèves ne semblent pas encore détenir de connaissances sur le concept de syllabes.

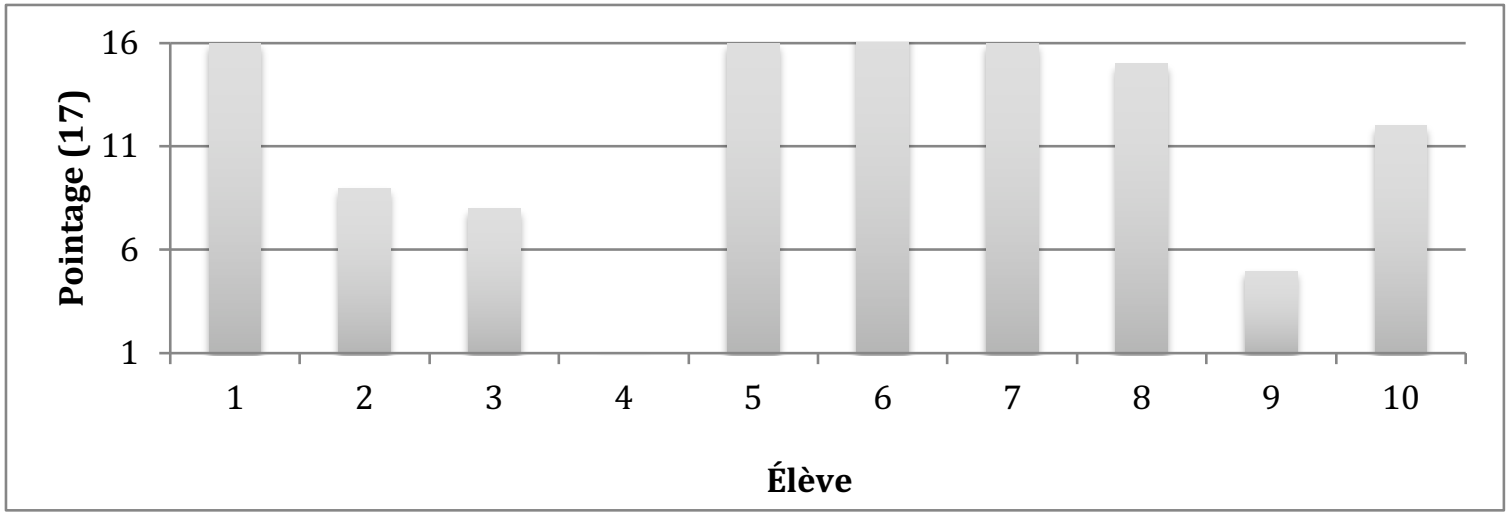

Figure 2. Score des élèves relativement à la connaissance et l'identification des syllables.

\section{Connaissances paratextuelles}

Cette activité s'intéressait aux connaissances que détiennent les élèves au regard des éléments présentés autour du texte. Précisément, nous cherchions à observer s'ils pouvaient identifier sur une première de couverture, le titre, l'auteur, l'illustrateur et la maison d'édition. 
Si une majorité d'élèves (6/10) a pu identifier le titre sur une première de couverture, il leur semble laborieux d'identifier l'auteur, l'illustrateur et la maison d'édition sur une première de couverture. Ici, on suppose que les parents, lors des pratiques de littératie familiales ou des occasions de lecture à l'enfant, ne s'attardent pas aux connaissances paratextuelles. Les conclusions semblent montrer que les parents, lors de leur lecture à l'enfant, s'arrêtent sur le titre, mais ne misent pas beaucoup sur la présentation de l'auteur, de l'illustrateur et de la maison d'édition.

\section{Principe alphabétique}

Relativement au principe alphabétique, nous cherchions à décrire les connaissances des élèves au regard de la linéarité d'écriture et de la reconnaissance de mots en français et en espagnol.

Linéarité d'écriture. Aux pages 6 et 7 du livret, nous cherchions à documenter les connaissances des élèves relatives à la linéarité d'écriture. À la page 6 , une page du livre $J e$ suis Louna et je suis une artiste où les élèves devaient, avec leur doigt, nous montrer dans quel sens se fait la lecture. L'activité de la page 7 différait légèrement de la page 6 dans la mesure où les élèves devaient indiquer la direction dans laquelle se fait la lecture lorsqu'il y a deux pages.

Ici, les sujets ont tous su indiquer avec leur doigt le sens dans lequel se fait la lecture. Que ce soit lorsqu'il y a du texte sur une page ou sur deux pages, les élèves ont su montrer que la lecture se fait de gauche à droite, du haut vers le bas. Au regard de cette conclusion, on peut supposer que le matériau écrit qu'est le livre est connu par les élèves de notre étude. En sachant que l'implication parentale joue un rôle fondamental dans l'apprentissage du lire-écrire (Kassow, 2006) et que les élèves ont su reconnaître le sens dans lequel se fait la lecture, nous pouvons supposer, là aussi, que les parents de notre étude faisaient la lecture à leur enfant. Il ne faut pas oublier non plus que les activités littéraciques dans le cadre scolaire ont certainement contribué aux connaissances des élèves sur le principe alphabétique.

En résumé, et selon nos interactions auprès des élèves de première année alors qu'ils remplissaient le livret, on peut facilement conclure que ceux-ci avaient une bonne connaissance du principe alphabétique. En effet, l'ensemble des élèves a su bien indiquer le sens dans lequel se fait la lecture et a démontré de bonnes connaissances paratextuelles (localisation du titre, de l'auteur et de la maison d'édition sur une première de couverture). Relativement au code alphabétique, certains enfants ont eu de la difficulté à reconnaître et identifier les syllabes. En fait, si plusieurs pouvaient décrire et définir ce qu'est une syllabe, c'est-à-dire un regroupement de deux ou de plusieurs lettres qui forment un son, ils n'ont toutefois pu les identifier correctement. Pour plusieurs, des mots et des lettres ont été encerclés en supposant qu'il s'agissait de syllabes.

Pour faire le point sur les connaissances initiales des élèves, il semble que ceux-ci « maîtrisaient » davantage les concepts macros qui appartiennent à la langue, soit la connaissance des lettres, des allographes et la linéarité d'écriture. À cet égard, l'on peut supposer que lors de la lecture aux enfants, les parents ne s'attardaient qu'aux éléments les plus caractéristiques de la langue. À l'inverse, les concepts plus micros, c'est-à-dire les syllabes, les phonèmes et les éléments paratextuels semblaient incompris ou totalement inconnus chez les élèves de notre étude. 


\section{Buts et fonction de la langue}

Le livret destiné aux enfants comportait aussi deux questions qui cherchaient à documenter leur connaissance des buts et des fonctions de la langue : 1) ça sert à quoi lire? ; 2) ça sert à quoi écrire. Aucun point n'était attribué aux réponses des élèves. Plutôt, nous avons enregistré l'entretien avec un appareil audio, dans le but de retranscrire les données de sorte à mieux les coder et les analyser.

Utilité de la lecture. Relativement à l'utilité de la lecture, tous les élèves ont mentionné certaines fonctions de la langue ce qui souligne qu'ils possédaient des connaissances initiales du rôle de l'écrit. Si certains ont expliqué que la lecture permettait de comprendre un message, d'autres ont mentionné qu'elle permettait plutôt de réaliser des tâches dans d'autres domaines connexes, par exemple, faire des mathématiques. Voici quelques exemples.

Chercheure: Et ça sert à quoi lire? Pourquoi on lit?

Ignacio: $\quad$ Pour apprendre

Chercheure: Pour apprendre, ok. Pour apprendre quoi par exemple?

Ignacio : $\quad$ Apprendre à lire parce que sinon, si on a un enfant et nous on est un papa, l'enfant va dire " qu'est-ce que ça veut dire ça?" Et moi je le sais pas.

Dans cet extrait de verbatim, Ignacio a souligné clairement l'importance de lire pour pouvoir se comprendre et avoir les mêmes outils pour communiquer. Donc cela représente à la fois un but et une fonction de la langue : la communication.

Chercheure : Alors à quoi ça sert lire? Pourquoi est-ce qu'on lit?

Dario : $\quad$ Pour apprendre

Chercheure: Ok, apprendre quoi?

Dario : $\quad$ Ça sert d'apprendre à compter, comme des mathématiques. Comme $4+4$ ça fait 8.

Dans l'interaction avec Dario, on parle de connaissances nouvelles pour, on imagine, être capable d'être autonome. Celui-ci a souligné l'importance des mathématiques.

Chercheure: Pourquoi est-ce qu'on lit?

Tamara: $\quad$ Pour savoir c'est quoi qui fait le personnage dans un livre

Chercheure: Ok. Est-ce qu'on lit pour autre chose?

Tamara : $\quad$ Euh, je ne sais pas

Ici, si Tamara comprenait que la lecture permettait de saisir les actions d'un personnage dans une histoire, elle ne semblait pas encore connaitre les buts et les fonctions de la langue écrite. Compte tenu de son âge et de son niveau scolaire, on peut aussi penser que Tamara focalisait sur le schéma narratif qu'elle était en train d'apprivoiser. 
Utilité de l'écriture. Sur le plan de l'écriture, les réponses des élèves en lien avec les buts et fonctions de la langue étaient généralement associées à des activités qu'ils pratiquent au quotidien à l'écrit. Par exemple, Ignacio a mentionné que l'écrit lui servait à écrire des cartes à ses parents tandis qu'Amal a indiqué que ça servait à écrire des mots à ses amis. Tamara, pour sa part, a mentionné que l'écrit lui servait à écrire une liste de cadeaux au Père Noël. Quelques exemples sont présentés ci-dessous.

Chercheure: Et maintenant, ça sert à quoi écrire?

Amal : $\quad$ Faire des cadeaux pour maman et papa

Chercheure: Ok, quel genre de cadeaux pour maman et papa?

Amal : $\quad$ Comme faire une carte

Pour Amal, l'utilisation de l'écrit pour l'enfant fait référence à un support - la carte. L'élève a démontré alors une connaissance du but de l'écrit - écrire une carte - et de son rôle - donner un cadeau à ses parents.

Chercheure : Ça sert à quoi écrire maintenant?

Ignacio : $\quad$ Écrire c'est pour, pour, écrire c'est comme dessiner en même temps, mais écrire c'est comme, si tu veux faire un message à l'ami, tu écris des mots parce que si tu écris n'importe quoi, l'ami ne va pas comprendre c'est quoi.

Chercheure : Ok, alors ça sert à écrire des mots à un ami. Est-ce que ça sert à autre chose? Ignacio : Non

Dans l'extrait de verbatim qui précède, on remarque que la participante a tenu compte du dessin pour expliquer l'utilité de l'écriture, en exprimant sa limite. Ici, l'élève savait que les mots permettaient au destinataire de comprendre le message, de quoi il était question.

Notre recherche nous permet aussi de faire un lien entre les connaissances des élèves au regard des syllabes et des buts et les fonctions de la langue. Précisément, nos données montrent que les élèves qui savaient identifier les syllabes détenaient également de bonnes connaissances quant aux buts et aux fonctions de la langue. À l'inverse, les élèves de notre étude qui détenaient de moins bonnes connaissances vis-à-vis des syllabes ont aussi présenté de moins bonnes connaissances face aux buts et aux fonctions de la langue. $\mathrm{Si}$, à notre connaissance, aucune n'étude ne s'est préalablement penchée sur la relation entre ces deux éléments langagiers, nous pouvons supposer, pour notre part, que les élèves qui ont présenté un score plus élevé à ces deux activités soient plus exposés au lire-écrire. Si nous savons que les pratiques de littératie familiales et que la fréquence et la qualité des implications parentales ont un impact significatif sur le développement des pratiques langagières (Leseman et de Jong, 2001 ; Sénéchal et LeFevre, 2002 ; Sénéchal, 2006), nous pouvons supposer que ces élèves pratiquaient souvent la lecture à la maison et que les parents offraient un soutien familial effectif.

En somme, nos résultats nous permettent de mieux comprendre et décrire les connaissances que détenaient les élèves hispanophones de $1^{\text {re }}$ année relativement aux buts et aux fonctions de la langue. Précisément, en lien avec l'utilité de la lecture, les enfants ont mentionné une certaine fonction d'apprentissage, par exemple, que la lecture permettait de comprendre un message ou qu'elle permettait de faire des mathématiques. En écriture, les 
réponses des élèves étaient généralement associées à des activités qu'ils pratiquaient à l'écrit, comme rédiger une carte pour maman et papa ou encore comme écrire sa liste de souhaits au Père Noël. Si Chauveau (2002) a mentionné que les premières années de la scolarité étaient généralement relatives à l'apprentissage du code alphabétique et des fonctions du lire-écrire, il est intéressant de constater que les usages de l'écriture et la lecture chez les enfants de notre recherche étaient très fortement associés à des activités qu'ils pratiquaient au quotidien ; comprendre un message, interpréter un message écrit, rédiger un message à partir de pseudo-mots.

Brièvement, et à la lumière de ces résultats, nous remarquons qu'au regard du principe et du code alphabétique, les élèves hispanophones de première année scolarisés en Outaouais détenaient des connaissances bien différentes les uns des autres. De la même manière, si les buts et les fonctions de la langue semblaient connus chez nos participants, nous pouvons supposer que la découverte du livre était une démarche personnelle, car en dehors de l'école, il existait peu d'occasions de littératie.

\section{Conclusion}

Notre objectif était de décrire les connaissances initiales d'élèves hispanophones de première année scolarisés français langue de scolarisation, en Outaouais, au regard du code et du principe alphabétique. Notre recherche nous permet d'affirmer que les élèves hispanophones de première année détenaient une certaine connaissance du principe et du code alphabétiques. Relativement au code alphabétique, les élèves savaient reconnaître et identifier les lettres et les allographes, mais ne comprenaient pas encore le concept de syllabes. En lien avec les buts et les fonctions de la langue, la majorité des élèves participants ont su décrire l'utilité du lire-écrire en associant, de manière tout à fait normale, ces fonctions à des activités qu'ils pratiquaient au quotidien.

En contexte scolaire, les résultats nous permettent de proposer des pistes didactiques et pédagogiques qui ont le potentiel de favoriser la réussite scolaire des hispanophones. D'une part, si nous savons que « [...] la substitution de la langue d'origine par la langue d'accueil risque d'avoir des conséquences négatives » (Mc Andrew et Ciceri, 2003, p.17), peut-être faudrait-il modifier les pratiques des enseignants de manière à encourager l'utilisation des langues d'origine des apprenants. D'autre part, si les recherches de Lahire (1995-2012) ont démontré que la famille et que l'école étaient des réseaux d'interdépendance structurés et que la réussite et l'échec scolaire dépendaient grandement de la relation entre ces réseaux, le contenu scolaire devrait davantage refléter la réalité des hispanophones. Ce sont, pour Castellotti et Moore (2010, p.17), dans de telles circonstances de rapprochement entre le milieu social familial et scolaire de l'apprenant qu'il sera possible de «tisser des liens avec les familles. »

Il serait intéressant, dans de futures recherches, de suivre le parcours scolaire des élèves de notre étude et de documenter le développement de leurs compétences relatives au français de scolarisation, notamment en fonction de leur participation à un programme de littérature de jeunesse. Considérant ceci, on assiste, depuis plusieurs décennies, à l'omniprésence des programmes de littérature de jeunesse pour soutenir l'apprentissage des élèves. Dans une perspective interculturelle, et sachant que l'utilisation de la L1 a une influence positive sur l'apprentissage de la L2 (Cummins, 2011), les livres de jeunesse favorisent l'apport du plurilinguisme et la prise en compte d'une diversité linguistique et culturelle, par exemple en sensibilisant les élèves quant à la diversité des langues, des 
cultures et des contextes, et en favorisant le dépassement des dichotomies traditionnelles entre langues et cultures (Moore et Sabatier, 2014). Sur le plan langagier, vu la multitude de choix, de formats et de contenus, cette littérature influence positivement la construction des répertoires langagiers des élèves et permet une meilleure compréhension des buts et des fonctions de la langue écrite.

La correspondance devrait être addressé à Marie-Pier Bastien.

Courriel : mariepierbastien@hotmail.com

\section{Notes}

${ }^{1}$ Les classes d'accueil sont des classes à effectif réduit dont l'objectif consiste à favoriser le développement d'habiletés langagières des élèves pour qu'ils puissent atteindre un niveau de scolarisation similaire à celui des élèves québécois (Armand, 2011).

${ }^{2}$ Bien qu'elles visent elles aussi l'apprentissage du français, les classes de francisation diffèrent des classes d'accueil dans la mesure où les élèves sont retirés de leur salle de classe, à des moments précis dans la semaine, pour recevoir un soutien additionnel en français (Armand, 2011).

${ }^{3}$ Par définition, la langue d'origine « désigne [...] un dispositif (non obligatoire) d'enseignement particulier, réservé aux enfants issus de l'immigration. Par extension, elle renvoie aussi aux idiomes avec lesquels ces enfants sont censés être en contact au sein de leur famille, même s'il existe des décalages importants entre les langues officielles enseignées et les vernaculaires en usage dans les familles migrantes » (Cuq et Gruca, 2003, p.153). Cette langue d'origine fait aussi référence au concept de langue maternelle, mais nous retenons le vocable langue d'origine puisqu'il est moins axé sur la mère (Auger, 2007).

${ }^{4}$ Brièvement, et parce que nous en discutons plus loin dans ce texte, les capacités métalinguistiques font référence aux connaissances métaphonologiques, morphologiques, syntaxiques et sémantiques (Fleuret, 2008).

\section{Références}

Armand, F. (2005). Capacités métalinguistiques d'élèves immigrants nouvellement arrivés en situation de grand retard scolaire. Revue des sciences de l'éducation, 31(2), 441469.

Armand, F. (2011). Synthèse des portraits de huit écoles primaires et de cinq commissions scolaires francophones de la région du grand Montréal (2007). Rapport de recherche sur le programme d'accueil et de soutien à l'apprentissage (PASAF) dans la région du grand Montréal.

Auger, N. (2007). Enseignement des langues d'origine et apprentissage du français: vers une pédagogie de l'inclusion. Le français aujourd'hui, 158(3), 76-83.

Burns, S., Espinosa, L., et Snow, C. (2003). Débuts de la littératie, langue et culture: perspective socioculturelle. Revue des sciences de l'éducation, 19(1), 75-100.

Castellotti, V. et Moore, D. (2010). Valoriser, mobiliser et développer les répertoires 
plurilingues et pluriculturels pour une meilleure intégration scolaire. L'intégration linguistique et éducative des enfants et des adolescents issus de l'immigration, (4), 125.

Citoyenneté et Immigration Canada. (2014). Faits et chiffres 2012 - Aperçu de

l'immigration : Résidents permanents et temporaires. Repéré à

http://publications.gc.ca/site/archiveearchived.html?url=http://publications.gc.ca/collections/collection_2013/cic/Ci1-82012-fra.pdf

Cummins, J. (1979). Cognitive/Academic Language Proficiency, Linguistic Interdependence, the Optimum Age Question and Some Other Matters. Working Papers on Bilingualism. Working Papers on Bilingualism, 19, 9.

Cuq J.P. et Gruca, I. (2003). Cours de didactique du français langue étrangère et seconde. Grenoble, Presses Universitaires de Grenoble.

Dagenais, D. (2012). Littératies multimodales et perspectives critiques. Recherches en didactique des langues et des cultures. Les cahiers de l'Acedle, 9 (2), 15-38.

Delgado-Gaitan, C. (2005). Family Narratives in Multiple Literacies. Anthropology \& Education Quarterly, 36(3), 265-272.

Fijalkow, J. (2003). Pourquoi et comment articuler l'apprentissage de la lecture avec celui de la production d'écrit aux différentes étapes de la scolarité primaire. Consensus sur l'enseignement de la lecture à l'école primaire. Paris : PIREF.

Fleuret, C. (2008). Portrait du développement de la compétence orthographique d'élèves créolophones scolarisés au Québec de la maternelle à la troisième année. [thèse de doctorat, Université de Montréal, Canada].

Gamez, P., et Levice, S. (2013). Oral Language Skills of Spanish-Speacking English Language Learners: The impact of High-Quality Native Language Exposure. Applied Psycholinguistics, 34, 673-696.

Gauthier, B. (1992). Recherche sociale: De la problématique à la collecte des données. Sillery, Québec: Presses de l'Université du Québec.

Hornberger, N. H. (2003). Continua of biliteracy: an ecological framework for educational policy, research, and practice in multilingual settings. Clevedon ; Buffalo, NY : Multilingual Matters.

Jiménez, C. Terese. (2006). Shared Reading Within Latino Families : An Analysis Of Reading Interactions and Language Use. Bilingual Research journal, 30(2), 431-452.

Kanouté, F., Vatz Laaroussi, M., Rachédi, L. et Tchimou Doffouchi M. (2008). « Familles et réussite scolaire d'élèves immigrants du secondaire. » Revue des sciences de l'éducation, 34(2),265-289.

Karsenti, I. et Savoie-Zajc, L. (2011). La recherche en éducation (3e éd.). Saint-Laurent (Québec) : Éditions du Renouveau Pédagogique (ERPI).

Kassow, D. (2006). Parent-Child Shared Book Reading Quality versus Quantity of reading Interactions between Parents and Young Children. Talaris Research Institute, 1(1), 19.

Lahire, B. (1995). Le point de vue de connaissance. Dans Tableaux de familles: Heurs et malheurs scolaires en milieux populaires (p. 29-63). Paris : Seuil/Gallimard.

Le Ferrec, C. (2008). Littératie, relations à la culture scolaire et didactique de la lecture écriture en français langue seconde. In J.-L. Chiss (Eds.). Immigration, École et didactique du français. (p.101-145). Paris, France : Didier.

Le Ferrec, L. (2012). Le français de scolarisation au carrefour des didactiques du français et 
des disciplines. Le français aujourd 'hui, 1(176), 37-4

López L., et Greenfield, D. (2004) The Cross-Language Transfer of Phonological Skills of Hispanic Head Start Children. Bilingual Research Journal, 28(1), 1-18.

Leseman, M-P. et De Jong, P. F. (1998). Home literacy : Opportunity, instruction, cooperation and social-emotional quality predicting early reading achievement. Reading Reasearch Quaterly, 33(3), 294-318.

Mc Andrew, M., Garnett B., Ledent, J., Ungerleider, C., Adumati-Trache, M. et Ait-Said, R. (2009). La réussite scolaire des élèves issus de l'immigration : une question de classe sociale, de langue ou de culture ? Éducation et francophonie, 36(1), 177-193.

Mc Andrew, M. et Ciceri, C. (2003). L'enseignement des langues d'origine au Canada : réalités et débats. Revue européenne des migrations internationales, (19)(1), 173-194.

Ministère de l'Éducation, du Loisir et du Sport. (2014). Accueil et intégration des élèves issus de l'immigration au Québec. Repéré à http://www.education.gouv.qc.ca/references/publications/resultats-de-larecherche/detail/article/accueil-et-integration-des-eleves-issus-de-limmigration-auquebec-entrevue-initiale/

Saracho, O. (2007). Hispanic Families as Facilitators of Their Children's Literacy Development. Journal of Hispanic Higher Education, 6(2), 103-117.

Sénéchal, M. et Lefèvre, J-A (2002). Parental Involvement in the Development of. Children's skills : A Five-Year longitudinal. Study. Child Development, 73(2), 445460.

Sénéchal, M. (2006). Testing the Home Literacy Model : Parent Involvement in Kindergarten Is Differentially Related to Grade 4 Reading Comprehension, Fluency, Spelling, and Reading for Pleasure. Scientific Studies of Reading, 10(1), 59-87.

Spaëth, V. (2008). Le français «langue de scolarisation» et les disciplines scolaires. Dans J.-L. Chiss (Eds.). Immigration, École et didactique du français (p.62-100). Paris, France : Didier.

Statistiques Canada. (2012). Définition des régions métropolitaines de recensement (RMR) et agglomérations de recensement $(A R)$. Repéré à https:/www150.statcan.gc.ca/n1/pub/92-195-x/2011001/geo/cma-rmr/cma-rmrfra.htm

Verdelhan, M., Maurer, B. et Durand, M-C. (1999). Le français, langue de scolarisation: vers une didactique spécifique. Tréma, 15(16).

Verdelhan-Bougrade, M. (2002). Le français de scolarisation : pour une didactique réaliste. Paris : Presses universitaires de France.

Vernon, S. et Ferreiro R. (1999) Writing Development: A Neglected Variable in the Consideration of Phonological Awareness. Harvard Educational Review, 69(4), p.395-416.

Vernon, S., Calderón, G. et Castro, L. (2004). The relationship between phonological awareness and writing in spanish speaking kindergartens. Written Language and literacy, 7(1), 101-118.

Ville de Gatineau. (2013). L'immigration à Gatineau de 1800 à 2010. Service des arts, de la culture et des lettres. Repéré à http://www.gatineau.ca/docs/guichet_municipal/immigration_diversite_culturelle/i mmigration_1800_2010.fr-CA.pdf 
Volk, D. (1998). «The teaching and the enjoyment and being together...»: Sibling teaching in the family of a Puerto Rican kindergartener. Early Childhood Research Quarterly, 14(1), 5-34. 